\title{
Collegiate Recovery Program (CRP): Student Needs and Employee Roles
}

Nika Gueci, MA

Arizona State University

\begin{abstract}
Background: Research on Collegiate Recovery Programs (CRPs) is emerging. There is a need to examine aspects of CRPs to highlight program efficacy and best practices within local settings.

Aim: As distinct cycles within a larger action research study, two small inquiries within a local university setting assessed: (1) how student members of the organization "Students for Recovery" defined their needs, and (2) how student employees of the CRP described their roles and success in their work.

Methods: Semi-structured interviews examined students in recovery and student CRP employees.

Results: Thematic analysis for each group produced two sets of qualitative results. Members of the student organization "Students for Recovery" described their needs as social support and safety, awareness and advocacy. Student employees of the CRP described their role as outreach and one-on-one support as well as strategic program planning. Instances of success were described as the departmental support, and their unique peer perspective.

Conclusions: Two queries described needs of students in recovery and role descriptions of student CRP employees within a specific setting. Robust studies are needed to understand the impact of local CRP programs and can provide context to guide informed, responsive programming and best practices.
\end{abstract}

Submitted 29 April 2018: accepted 5 September 2018

Keywords: Collegiate Recovery Program (CRP), college health, substance use and misuse, addiction recovery

The need for substance use and misuse recovery support services has never been higher. Nationally, total drug overdose rates have increased 211\% since 1999 (Center for Disease Control, 2017). From 1999 to 2015, prescriptions for opioid medication quadrupled, leading to six out of 10 drug overdose deaths involving opioids (Center for Disease Control, 2017). Deaths from prescription painkillers have reached epidemic proportions, with 33,091 people dying from opioid use and misuse in 2015 (Center for Disease Control, 2017). Alcohol use and misuse continues to be a leading cause of morbidity and mortality in the United States, accounting for one in 10 deaths in 20-64-year-old working adults, responsible for 88,000 deaths from 2006 - 2010 (Center for Disease Control, 2014).

The task of tackling addiction and providing supportive structures is a massive undertaking within the larger environment of a national drug epidemic (Cadeau, 2016; U.S. Department of Health and Human Services, 2016; Logan, 2017; National League of Cities \& National Association of Counties, 2016; Wydale, 2016). The objectives of former President Barack Obama's National Drug Control Policy were to prioritize and address the issues of substance abuse disorder through de-stigmatization, enhanced medical services, support services for recovery to non-violent drug offenders, and enhanced efforts at prevention of first alcohol and other drug use (Office of Drug Control Policy, 
2014). Yet while there have been moves made nationally related to drug policy, prevention, and recovery support for the general public, there are specific concerns for the college-aged student population. As college students are in a unique stage of development both psychologically and emotionally, programs that focus on drug or alcohol interventions must be developed with this population's particular needs in mind.

Collegiate Recovery Programs (CRPs) were developed to provide support to students in recovery from alcohol and other drug addiction and are intended to create a safe space in what is often thought of as an "abstinence hostile" university environment (Cleveland, Harris, Baker, Herbert, \& Dean, 2007). While current research exists on the need for CRPs (Laudet, Harris, Kimball, Winters, \& Moberg, 2014; Smock, Baker, Harris, \& D’Sauza, 2011), the student experience of recovery (Worfler, 2016), and descriptions of local CRP models (Beeson, Whitney, \& Peterson, 2017), "much more research is needed to illuminate efficacy and the most effective mechanisms for CRPs" (Holleran Steiker, Grahovac, \& White, 2014) as well as the "need for continued research regarding the impact of entire programs as well as individual programmatic elements" (Beeson et al., 2017). These research recommendations provide incentive to offer insight into effective practices through examination of programmatic elements within individual CRPs.

This article reports on two cycles of action research conducted around CRPs in a university context. The first cycle is intended to understand the needs of students in recovery in order to create services that best meet those needs. The second cycle investigates how student employees of the CRP view their role as providers of recovery support to their peers. Both cycles serve to supply information on programmatic elements as well as create a foundation of understanding for the larger action research dissertation study.

\section{BACKGROUND}

The college-aged population is unique from the adult population at large, especially as related to alcohol and other drugs. Developmental models may help explain why some students have a greater or lesser propensity for psychological distress and adverse health outcomes in college. One such model is the overload model (Schulenberg \& Maggs, 2002), which describes how many developmental transitions (i.e., unfamiliar environment, new friendships, challenging coursework) experienced all at once can lead to stress. Some students may be at an increased health risk in college due to peer influence or environmental factors and turn to alcohol or other drugs to cope with stress (National Institute on Alcohol Abuse and Alcoholism, Task Force of the National Advisory Council on Alcohol Abuse and Alcoholism, 2002; Schulenberg \& Maggs, 2002).

Late teenage years and early adulthood is a time some individuals perceive to be their "drinking years" (Schulenberg \& Maggs, 2002, p. 54). Relationships (e.g., family, friends, or school personnel) and daily environments (e.g., home, school, or friend's houses) play a role in how teenage and young adults develop stress coping responses and in turn, affect their perception of and relationship to alcohol and other drugs. These developmental networks influence drinking or non-drinking patterns. Generally, these social and familial relationships and environments provide support and context to the growing individual. Transitions (i.e., the move from family home) affect individuals to greater or lesser degrees (Schulenberg \& Maggs, 2002). The transition from high school to college is one such stressor that can lead to the development of unhealthy methods of coping, such as alcohol use.

Adaptation between one system and another is an important factor in this transition. Risk factors (e.g., such as genetic predisposition to substance abuse) and protective factors (e.g., strong social support networks) are key to note here as well. The effects of the transition from high school to college is seen as a determination point where students pick up healthy or unhealthy behaviors and habits which continue on to their adult years. In this way, college is a huge 
determinant of health and wellness (Schulenberg \& Maggs, 2002). Healthy social connection can provide a protective barrier against the risk of peer pressure and influence.

Collegiate Recovery Programs (CRPs) were specifically developed for the college-aged population. Within the university environment, CRPs provide social support as a prevention strategy to relapse (Smock et al., 2011). The challenges encountered in residence halls can be unique to the student population, who are generally younger aged and do not have families to support. As such, the difficulties of becoming alcohol- and drug-free and maintaining sobriety are different for a college student than for an older adult. College campuses have long been perceived as environments that are ripe for substance use and abuse, however, there exists a population of students who are in recovery from addiction. This perception of an unsupportive environment on campus can be detrimental to student recovery. When students do not feel supported in their social, academic or personal lives, they have difficulty persisting and thriving in college (Nicpon et al., 2006; Solberg \& Viliarreal, 2016). For students in recovery, support in the form of abstinence and recovery maintenance is necessary. CRPs facilitate a culture of social engagement and support, which can go a long way in sustaining individual and community well-being for students in recovery.

Students who join CRPs note that their participation serves as a barrier between themselves and the potentially drinking-heavy culture of the university environment, and grants a valuable method to finding a like-minded community of their peers in the college campus space where they feel otherwise alienated (Terrion, 2012). The "instant friendships" formed within the context of the CRP community protect students in recovery from isolation, which is linked to risk of relapse (Bell et al., 2009).

In an effort to meet the needs of students in recovery at a local level, a large public university in the Southwest (70,000+ students) began to develop a CRP in 2016. This university already offered students a range of support structures to meet well-being needs, including Counseling, Disability and Health service departments. Programs existed to provide students with extracurricular support as well, from student clubs to fun, free, and sober weekend activities. While such programs engaged students in socialization opportunities with their peers and promoted overall wellness, there was no formal institutional support program for students in recovery from alcohol or other drug addiction.

Within this local university setting, developing recovery support at the institutional level required the design of an effective CRP model. In order to be efficacious toward the goal of supporting student recovery, the CRP had to be responsive to student needs. Therefore, the first stage of programmatic development involved gathering information from students in recovery about how the CRP could best support their needs. The first cycle of action research described in this article sought to collect qualitative interview data from student participants involved in the organization "Students for Recovery" to determine how the CRP could be of assistance to meeting their recovery needs.

The second cycle of action research focused on understanding the perspective of student employees of the institutional CRP. As student CRP employees are on the front lines of working with their peers in recovery, identifying their beliefs and understandings about their role was also critical. Thus, qualitative data was gathered in order to better understand how they view their roles and how this contributes to the functioning of the CRP on campus.

The foundational data from these two small inquiries, along with university administrator input, led to the establishment of four goals for the CRP: To increase the visibility of the recovery lifestyle, to connect students in recovery to each other through meetings, sober activities and events, to enhance the ability of staff, faculty, and 
student leaders to support students who are in recovery through training opportunities, and to provide a continuum of care for students in recovery across campus departments.

\section{METHODS}

Two small qualitative inquiry methods for two different cycles of research within a larger action research project are described here. Action research involves collaboration within the institution of the researcher (Herr \& Anderson, 2015). The goal of action research is to improve practice within the setting of the researcher, as "action research is not the simple implementation of predetermined answers to educational questions; it explores, discovers, and works to find creative solutions to educational problems" (Mertler, 2014, p. 21). Action research calls for several cycles of investigation into a problem, implementation of a proposed solution, data collection and review. Both cycles of inquiry were conducted to understand how to create an institutionally relevant CRP model within the situational context. For the first cycle, members of the organization, "Students for Recovery" were interviewed. This inquiry was driven by the following research questions:

Research Question 1: How do students in the ASU organization "Students for Recovery" envision this group functioning?

Research Question 2: What kind of preparation or training would benefit "Students for Recovery" members as they work toward building their three organizational pillars of Awareness, Fun, and Service?

The second cycle of qualitative inquiry was related to how student CRP employee view their roles as providers of recovery support to their peers. This query was driven by the following research questions:

Research Question 1: How do student employees of the CRP describe their individual role?

Research Question 2: How do student employees of the CRP describe instances of success within their work?

The methods used to conduct research were semi-structured interview questions which were recorded using an audio-recorder, secured in a locked drawer, and transcribed without processing software by hand by the researcher. Each cycle of the study received Institutional Review Board (IRB) approval. For the first cycle, a recruitment letter was emailed to all the members of "Students for Recovery." Their email list was obtained during a previous meetand-greet interaction earlier in the semester. Three students replied to the request for interviews via email. A consent form was distributed to all students at the time of the interviews. The interviews lasted for approximately 20 minutes each. For the second cycle, participants were recruited in-person. A consent form was distributed to all studentemployees at the time of the interviews. The interviews lasted for approximately 30 minutes each.

The interview questions for each study were analyzed, and open coded line-by-line by hand to gather larger thematic data. After each cycle, the data was read for the main concepts and those concepts were analyzed into units of meaning, or "clusters of text that carry one meaning" (Koro-Ljungberg, Yendol-Hoppey, Smith, \& Hayes, 2017). The meaning units were identified by invivo codes in the text of the transcribed interviews. "Needs of students in recovery" and the "CRP student roles" were labeled, respectively, for each inquiry. The meaning units for each cycle were open coded and transferred to an Excel document where themes were identified. The method of thematic coding was chosen to find overarching concepts, ideas and phrases from the data sources in order to create key themes 
(Saldana, 2009). A thematic analysis of the data is presented in the next section. The data from cycle one and cycle two of action research was then brought together for further analysis and points of comparison or differentiation.

\section{RESULTS}

The first cycle of inquiry was conducted to generate knowledge on the needs of students in recovery for the purpose of creating a responsive CRP within a local university setting. Three undergraduate participants from the student organization, "Students for Recovery" (i.e., two males, one female, all between 18 and 24 years of age) were interviewed. They were all in recovery from drug and alcohol addiction as well as working toward an undergraduate degree, which were the requirements for membership within the organization. This participant sample was chosen because they were members of the only student organization on campus that focused on recovery. The student organization was formed in 2015 and the interviews were held in 2016, therefore, the organization was in its nascent stages of development. The pillars that guided the organizational activities were: Awareness, Fun, and Service.

Several key themes emerged from the data, including: Social support/safety, education and awareness, and advocacy.

To address research question (RQ) \#1, all participants made explicit the importance of social support as a function of their groups, "Students for Recovery." When asked the question, "what is the function of the group?" all had similar answers: "To have a safe, fun place for students in recovery"; "as a sober alternative to stereotypical college life," and "this is a support for students in recovery that are on campus." The idea of safety was expressed, but importantly, students seemed to understand this idea not as safety from harm, rather, safety to be oneself without judgement. One participant mentioned that their group was the "safe spot" for them on campus and another expressed a desire to see a dedicated, physical space for the group: "Ideally, it would be nice to have a safe place on campus." This indicates the importance and value of the potential role of CRPs as offering students a place where they can feel safe in being and expressing themselves, without the external pressures they might feel otherwise.

To address RQ \#2, "what kind of preparation do you need to work effectively with students?" the theme of education and awareness or advocacy emerged. Students need to have education about what it means to be in recovery was expressed by all three participants: "recognizing alcoholism and drug addiction as a disease and not a choice and to not hold it against you, to not be put at a disadvantage," and "understanding exactly what addiction is: partying too much is not addiction." To work more effectively with their peers, an awareness of the existence of the student organization is necessary: "Awareness just that it [the group] exists, spreading the awareness"; "getting more members," and "making sure departments [on campus] know" about the group. A participant noted the importance of recognizing the resources for students in recovery, such as the Disability Center (DC), which may mitigate problems with classes or grades. For one participant, registering with the DC was a form of protection against negative outcomes, such as failing grades due to being absent.

Advocacy was listed as strength and future direction of the group. One participant noted that the campus was a "recovery desert," with minimal recovery meetings, and stated that "meetings on campus" would be "ideal." Another participant stated, "I would hope that...the club does sporting events together, advocate, get bigger." When asked to describe the strengths of the group, participants said, "the fact that people have the desire to start something from nothing," "fellowship," and "I want this thing to be a thing." Taken together this suggests that students in recovery feel the need for better educational supports on campus, and the potential value in CRPs as a site of education and awareness about issues of recovery and addiction. 
For the second qualitative inquiry, two students were interviewed (one undergraduate, one graduate, both male and between 18 and 24 years of age). This sample of participants had one criteria: employment within the CRP. The specifications of the participants as student employees provided a detailed description of their roles within the CRP. This inquiry focused on how student employees within the CRP described their role, the systematic method of identifying meaning units and coding produced four themes: outreach, one-on-one support, strategic planning as a function of the student role as employees of the CRP, and instances of success in their roles as providers of support.

To address RQ \#1, "how do student employees of the CRP describe their individual role?," the results indicated that both students described their main role as one of outreach ("reaching out to sober living houses and treatment centers"), one-on-one support ("intimate and personal one-on-one conversations") and strategizing the most effective way of "figuring out how two people [in the CRP] can reach [70,000 students]." Their outreach process included "educating more students and their peers in their community and residence halls." The role of having "one-on-one" conversations with student peers was identified as a benefit that "builds on a level of connectivity." Strategies to conduct the work required of the CRP included having to "figure out what is the best thing for that one individual student" and the "educated tactic" of using student survey data for "help" and "changing the cultural norm" of the college environment. These data suggest that student CRP employees view their role as one of delivering assistance to their peers in recovery directly (e.g., conversation and outreach) and indirectly (e.g., developing programmatic strategy). CRPs can strengthen efforts of student employees by making accessible institutional data to inform programmatic strategy.

To address RQ \#2, "how do student employees of the CRP describe instances of success within their work?" one participant stated, "this is one of the most difficult things I've ever done in my life, but also the most enjoyable," and noted a "supportive" office atmosphere. Another described that "staff has been very receptive of feedback from students and the community" which "led to very huge success in the program." The role of being a student rather than a staff member was noted as a success for both students which allowed them to have served a unique role. For instance, one person noted, "I know what it's like to have the pressures and the stresses of going through your undergrad" which "goes a long way...in terms of my relationships with my fellow students" and that a student in recovery is more likely to "reach out to you in order to share about their ideas." These findings suggest that a supportive CRP office setting and feedback from university staff can contribute to student employees' perception of success within their jobs. As noted above, CRP employees remark that their student status can be leveraged to better understand and relate to their

peers in recovery. Therefore, CRPs can potentially benefit from hiring student workers to connect with their peers in recovery.

\section{DISCUSSION}

These two inquiries were investigations into the needs of students in recovery followed by descriptions of student employee roles within the CRP. In the first cycle of action research, needs of students in recovery were defined in an effort to leverage the CRP to be in a position to better understand and support these students. In the second cycle of action research, student CRP employees defined their roles and instances of success within their work. These two cycles of introductory research informed and shaped the groundwork for the larger action research study of supporting students in recovery using a peer-to-peer CRP model of support.

Thematic results from the first cycle of inquiry suggest that students in recovery are interested in receiving social support and safety from those within their local environment, education and awareness about what addiction and 
recovery really means, and advocacy of a recovery lifestyle. One participant stated that the "Students for Recovery" organization was necessary to create because students in recovery "identified a need" on what was thought of as a campus "recovery desert." "Awareness," "reducing stigma" toward those in recovery, and "social support" were listed as idealized states of the group. Results suggest that increased services in the form of social support structures, addiction and recovery awareness and education, and advocacy for a recovery lifestyle would be beneficial to students in recovery. CRPs that provide a framework of social support and advocacy may reduce isolation and increase community, which is connected to relapse prevention, thriving and general well-being (Bell et al., 2009; Nicpon et al., 2006; Solberg \& Viliarreal, 2016; Terrion, 2012). Within a larger national setting where alcohol and drug use has reached epidemic proportions, CRPs have an opportunity to make a difference to those in recovery in their local context.

Thematic results from the second cycle of inquiry illustrate how student employees of the CRP define their roles and instances of success within their work. Student CRP employees describe their role as one of outreach to organizations and departments, supporting individual students in recovery through one-on-one conversations, and conceptualizing strategic program planning through the use of student trend survey data. Success within their roles was defined as the backing of a supportive department as their work environment, along with having the unique perspective of a student (rather than a staff member) to better understand their peers in recovery. Developmentally, college-aged students are a distinct population that is heavily influenced by peer relationships (Schulenberg \& Maggs, 2002). Therefore, local recovery services should account for the specific needs of this population by examining peerto-peer support structures.

The methods, data collection and analysis was similar for both studies. Results yielded information about both groups - students in recovery and student employees of the CRP. While there were similarities thematically, there were also some differences in terms of focus of the data, as two separate groups of students were interviewed.

Strategic planning as a programming tactic was not mentioned by students in recovery, perhaps because they did not have knowledge - or ready access to - institutional data on student trends. The student CRP employees, however, did have access to such data, and were therefore able to develop strategic programming approaches specific to their local context. Additionally, as "Students for Recovery" was a new organization, the research questions did not focus on their perceived successes, either individually or as a group. However, the research on the CRP student employees did allow for inquiry into perceptions of success within their jobs.

Despite some of these noted differences in the data, there were also key instances of alignment in ideas, which creates a broader picture of student needs as connected to student employee roles. For example, students in recovery noted a need for recovery advocacy, promotion and outreach, while the student CRP employees defined their role as one of outreach to departments and student organizations. Students in recovery stated that social support would be valuable, while student CRP employees represented their role as providers of one-on-one support. A broader picture of student needs and employee roles can advance understanding of local programmatic mechanisms and shorten current gaps in CRP research (Beeson et al., 2017; Holleran Steiker et al., 2014).

It is valuable to note that the relationship between the needs of students in recovery and peer support can be gauged by how the students employed by the CRP defined success in their jobs. Studying populations in need, such as students in recovery, alongside queries of those who are employed to support them, can provide information on best practices. If support services by student CRP employees match the call to action by their peers in recovery, then 
programmatic objectives are aligned with institutional goals, thereby bridging the gap between need and fulfilment of need.

\section{CONCLUSIONS, LIMITATIONS, \& IMPLICATIONS FOR FUTURE RESEARCH}

The purpose of these two cycles of qualitative inquiry was to integrate information learned locally and through my time leading the CRP with existing knowledge from the larger national context, in order to provide the most informed and responsive services for students in recovery. These small pieces of knowledge gained from both cycles of action research provided useful data on the needs of students in recovery and the ways in which student employees of the CRP view their roles and define success within those roles, respectively. Through the first cycle of inquiry, the needs of students in recovery were identified as a desire for social support and safety within the university environment, education and awareness of what it means to be in recovery (for those who are not in recovery, such as other students and faculty), and a desire to lead advocacy activities through their organization "Students for Recovery." In the second cycle of inquiry, the CRP student employee roles identified themselves as providers of outreach and one-on-one support, and strategic planners of the most effective methods to provide services to their peers in recovery. Instances of success within their role involved the receptivity and support of university staff, and having the unique perspective of a peer worker to better understand other students.

As part of a larger action research study, these two distinct cycles of research informed on the needs of students in recovery as well as the description of roles of student employees of the CRP. Taken together, the cycles provided context to the larger action research study and instruction on programmatic development of the CRP at an institutional level. The future of the CRP program within this local context will be further guided by the findings produced at the end of the larger action research study.

One obvious limitation is that this research is not generalizable, however standard generalizability was never a goal of this project--rather it was about developing a better understanding of needs and roles within a CRP at a local level, which importantly is the level at which CRPs have impact in varied types of institutions. Generalizability is not a goal of action research. Transferability, or the transferring of research findings from one context to similar situations or participants, is a goal of action research (Herr \& Anderson, 2015). CRPs exist within local environments, so it's important to find ways to understand key themes and consider their transferability to other institutional factors and culture - rather than seeking broad-scope generalizability - but to look at practices at the local level to see how they might apply in a different setting.

A limitation across both cycles of research is in the small sample size of five students, though small samples of "up to 10" are not uncommon in qualitative research (Creswell, 1998). This size limitation in the first cycle of research is reflected in the fact that it is a small-scale inquiry with only three participants from whom qualitative data was gathered. Further, because "Students for Recovery" was a newly formed organization at the time of the interviews, the questions that the students had been grappling with were still in the process of being determined (for example, whether this group would be open to all students in recovery - (i.e., from eating disorders or gambling) or whether the group would only allow those in recovery from drug and alcohol addiction). In time, the group will have answers to questions such as these, solidifying their stance on what resources to bring awareness to ("awareness" being one of their organizational pillars). As both the researcher and a staff member of the CRP, familiarity bias must also be noted. The invivo description of the student employee experience aimed to promote objectivity and reduce bias by examining the data using the direct words of the participant. 
Recommendations for future research involve the need for larger and more robust studies to explore what students in different universities require from a CRP. To understand local environments, and the distinct views of the students within those environments, further research can provide contextual information to guide institutional programs. Additionally, it may also be that through a greater number of small-scale local studies the area of recovery can benefit from making connections and applications across varied local settings.

This investigation into aspects of a local CRP connects to the broader picture of a need for institutions to understand the distinct needs of students in recovery so that they may provide the most comprehensive services to this population. 


\section{REFERENCES}

Beeson, E. T., Whitney, J. M., \& Peterson, H. M. (2017). The Development of a Collegiate Recovery Program: Applying Social Cognitive Theory within a Social Ecological Framework. American Journal of Health Education, 48(4), 226-239. https://doi.org/10.1080/19325037.2017.1317304

Bell, N. J., Kanitkar, K., Kerksiek, K. A., Watson, W., Das, A., Kostina-Ritchey, E., \& Harris, K. (2009). "It has made college possible for me": Feedback on the impact of a university-based center for students in recovery. Journal of American College Health, 57(6), 650-658. https:/ / doi.org/10.3200/JACH.57.6.650-658

Cadeau, C. (Host). (2016). Persevering with Joanna Sweatt [Radio series episode]. Veteran diaries. Phoenix, AZ: Blaze Radio Online.

Center for Disease Control. (2017). Opioid basics. Retrieved from https://www.cdc.gov/drugoverdose/opioids/ index.html.

Center for Disease Control. (2014). Alcohol deaths. Retrieved from https://www.cdc.gov/features/alcoholdeaths/index.html.

Cleveland, H. H., Harris, K. S., Baker, A. K., Herbert, R., \& Dean, L. R. (2007). Characteristics of a collegiate recovery community: Maintaining recovery in an abstinence-hostile environment [Electronic version]. Journal of Substance Abuse Treatment, 33, 13-23. https://doi.org/10.1016/j.jsat.2006.11.005

Creswell, J.W. (1998). Qualitative inquiry and research design: Choosing among five traditions. London: Sage.

Herr, K., \& Anderson, G. L. (2015). The action research dissertation: A guide for students and faculty (2nd ed.). Thousand Oaks, CA: Sage.

Holleran Steiker, L. K., Grahovac, I., \& White, W. L. (2014). Social Work and Collegiate Recovery Programs. Social Work, 59(2), 177-180. https://doi.org/10.1093/sw/swu012

Koro-Ljungberg, M., Yendol-Hoppey, D., Smith, J., \& Hayes, S. (2009). (E)pistemological awareness, instantiation of methods, and uninformed methodological ambiguity in qualitative research projects. Educational Researcher, 38(9), 687-699. https://doi.org/10.3102/0013189X09351980

Laudet, A., Harris, K., Kimball, T., Winters, K. C., \& Moberg, D. (2014). Collegiate Recovery Communities Programs: What do we know and what do we need to know? Journal of Social Work Practice in the Addictions, 14(1), 84-100. https://doi.org/10.1080/1533256X.2014.872015

Logan, S.P. (2017). HookedRX: From prescription to addiction. Tempe, Arizona: Walter Cronkite School of Journalism and Mass Communication.

Mertler, C. A. (2014). Action research: Improving schools and empowering educators (4th ed.). Thousand Oaks, CA: Sage.

National Institute on Alcohol Abuse and Alcoholism, Task Force of the National Advisory Council on Alcohol Abuse and Alcoholism. (2002). A call to action: Changing the culture of drinking at U.S. colleges. Retrieved from http://www.collegedrinkingprevention.gov/media/taskforcereport.pdf

National League of Cities (NLC) and National Association of Counties (NACo) (2016). A prescription for action: Local leadership in ending the opioid crisis. Washington DC: NLC and NACo. 
Nicpon, M.F., Huser, L., Blanks, E.H., Sollenberger, S., Befort, C., Kurpius, S.E.R. (2006). The Relationship of Loneliness and Social Support with College Freshmen's Academic Performance and Persistence. Journal of College Student Retention: Research, Theory \& Practice, 8 (3), pp. 345 - 358. https://doi.org/10.2190/A465-356M-7652-783R

Office of National Drug Control Policy. (2014). National Drug Control Strategy. Washington, DC: U.S. Government Printing Office.

Saldaña, J. (2009). The coding manual for qualitative researchers. Thousand Oaks, CA: Sage Publications Ltd.

Schulenberg, J. E., \& Maggs, J. L. (2002). A Developmental Perspective on Alcohol Use and Heavy Drinking during Adolescence and the Transition to Young Adulthood. Journal of Studies on Alcohol, 54(70), 54-70. https://doi.org/10.15288/jsas.2002.s14.54

Smock, S. A, Baker, A. K., Harris, K. S., \& D'Sauza, C.A.B. (2011). The Role of Social Support in Collegiate Recovery Communities: A Review of the Literature. Alcoholism Treatment Quarterly, 29 (1): 35-44. https://doi.org/10.1080/07347324.2010.511073

Solberg, V. S., \& Viliarreal, P. (2016, July 25). Examination of self-efficacy, social support, and stress as predictors of psychological and physical distress among Hispanic college students. Hispanic Journal of Behavioral Sciences, 19(2), 182-201. https://doi.org/10.1177/07399863970192006

Terrion, J. L. (2012). The experience of post-secondary education for students in recovery from addiction to drugs or alcohol: Relationships and recovery capital. Journal of Social and Personal Relationships, 30(1), 3-23. https://doi.org/10.1177/0265407512448276

U.S. Department of Health and Human Services (HHS), Office of the Surgeon General. (2016). Facing addiction in America: The Surgeon General's Report on Alcohol, Drugs, and Health. Washington, DC: HHS.

Worfler, K. R. (2016). Examining college students in recovery from a substance use disorder through interpretative phenomenological analysis (Order No. 10138968). Available from ProQuest Dissertations \& Theses Global. (1823220943). Retrieved from https://search.proquest.com/docview/1823220943.

Wydale, H.K. (2016). Opioid action planning: Collective actions to reduce opioid abuse and related fatalities in the region. City of Tempe, Arizona: Office of Strategic Management and Diversity.

Address author correspondence to:

Nika Gueci

Nika@asu.edu 


\section{APPENDIX A \\ FIRST CYCLE INTERVIEW QUESTIONS - STUDENTS IN RECOVERY}

1. What is the function of the group, "Students for Recovery"? What is the mission and vision of this group?

2. What is the future of this group? Where will "Students for Recovery" be in several years?

3. What kind of preparation do you need to work effectively and serve other students?

4. What are the strengths of the group?

5. What are the barriers that might prevent the group from achieving its full potential?

\section{APPENDIX B \\ SECOND CYCLE OF INTERVIEW QUESTIONS - CRP STUDENT EMPLOYEES}

1. You are a student employee of the Collegiate Recovery Program (CRP). Tell me about your work.

2. Describe the function of the CRP.

3. Tell me about how university administrative staff has responded to the CRP.

4. This is a university with over 70,000 students. Describe your role as a CPR student employee in serving those students.

5. Describe your role in supporting students in recovery from alcohol and other drug addiction at the university.

6. What are the benefits to having a peer employee in this role?

7. What are the obstacles to having a peer employee in this role?

8. Is there anything else you would like to add 